\title{
Selectivity of Macaque Inferior Temporal Neurons for Partially Occluded Shapes
}

\author{
Gyula Kovács, Rufin Vogels, and Guy A. Orban \\ Laboratorium voor Neuro-en Psychofysiologie, KULeuven, Campus Gasthuisberg, Herestraat, B-3000 Leuven, \\ Belgium
}

\begin{abstract}
Humans are able to recognize shapes even when they are partially occluded by another pattern. We determined whether inferior temporal (IT) units of awake rhesus monkey remain selective for shapes that are partially occluded by other patterns. In order to link the shape selectivity to shape discrimination, we established that the monkey, like humans, can discriminate partially occluded shapes. We found that IT units are selective for shapes partially occluded by static or moving patterns. The responsiveness, however, decreased with the degree of occlusion. The shape-selective responses to the occluded shapes required longer stimulus durations compared to nonoccluded shapes, matching the better shape discrimination performance at short durations in the no-occlusion compared to the occlusion conditions. Occluder visibility had no effect for shape outlines, indicating that (amodal) shape completion, which is absent when the occluder is invisible, is not necessary for shape selectivity. These data indicate that IT neurons can, indeed, contribute to the invariance of shape perception for changes in retinal input caused by partial occlusion of the shape.
\end{abstract}

[Key words: inferior temporal cortex, macaque monkey, shape perception, shape selectivity, shape occlusion, completion, visual cortex]

Single neurons in the inferior temporal (IT) corlex of the Indcaque monkey respond selectively to patterns differing in shape (Gross et al., 1972; Desimone et al., 1984; Tanaka et al., 1991). This shape selectivity is thought to underlie shape perception and recognition. Indeed, the properties of shape selectivity investigated so far match characteristics of higher shape perception. Shape recognition is relatively position (Biederman and Cooper, 1991), size (Biederman and Cooper, 1992), and visual cue invariant (Regan, 1991; Regan and Hamstra, 1991), and the shape selectivity of IT neurons generally is also invariant with changes in the position (Gross and Mishkin, 1977; Schwartz et

\footnotetext{
Received July 8, 1994; revised Sept. 6, 1994, accepted Sept. 12, 1994.

We are grateful to P. Kayenbergh, G. Meulemans, G. Vanparrijs, and A. Coeman for technical support. Dr. W. Spileers helped with the search coil surgery and M. De Paep's soft- and hardware made the computers run. We also thank Dr. S. Raiguel for correcting the linguistic errors. This research was supported by grants IUAP-22 Vision and Memory from the National Ministry of Science, FGWO 9.0039.90 from the Regional Medical Research Council and Human Capital and Mobility CHRX-CT93-0267 (DG12-COMA). R. Vogels is a research associate of the Belgian Fund for Scientific Research. Gy. Kovács was on leave from the Department of Physiology of the Albert SzentGyörgyi Medical University, Szeged, Hungary.

Correspondence should be addressed to Dr. R. Vogels at the above address.

Copyright (C) 1995 Society for Neuroscience 0270-6474/95/151984-14\$05.00/0
}

al., 1983), size (Sato et al. 1980; Schwartz et al. 1983; Rolls and Baylis, 1986), and the cue defining (Sáry et al., 1993) the shapes. In the present study, we determined whether the shape selectivity of IT neurons is invariant for changes in the retinal input caused by partial occlusion of the shape.

Imagine presenting a banana to a monkey sitting in its cage. Despite the fact that parts of the fruit are obstructed by the cage's bars, the monkey will surely recognize it, as will humans, as the reader can verify himself by looking at Figure 1 . Indeed, in everyday life, it is quite common that an object is partly occluded by intervening objects so that only parts of its shape are imaged on the retina. Nonetheless, one is able to recognize these partly occluded objects so easily that one is usually not even aware of the occlusion. Furthermore, physically disconnected parts of the shape are perceptually combined into a representation of a single form (a "Gestalt"), e.g., in Figure 1 one perceives a banana behind an occluder, and not a set of isolated banana parts together with the occluder. This type of completion of a partly occluded shape has been termed "amodal completion" by Michotte (1964), amodal because the occluded contours are not seen as differing in brightness from the occluder, in contrast to illusory contours (modal completion; Kanizsa, 1979; Michotte, 1964). These perceptual phenomena raise two questions regarding the shape selectivity of IT cells: (1) do IT units remain selective for shapes that are partly occluded by another pattern, and (2) do they represent completed shapes or simply disconnected shape elements?

We examined these questions by quantitatively comparing the responses of IT units in alert macaques to shapes presented with and without partial occlusion. Properties of both the occluder and the shape were varied in order to relate the response characteristics of the neurons to perception. Furthermore, in order to link the single unit properties of macaque IT to those of shape perception, we measured shape discrimination performance behaviorally under the same occlusion conditions as those used in the physiological experiments. These psychophysical experiments were performed with humans as well as with one of the monkeys used in the physiological recordings. Thus, we could demonstrate that monkeys as well as humans are able to discriminate the partially occluded shapes used in the single cell recordings.

Some of the results have appeared in abstract form (Vogels et al., 1993).

\section{Materials and Methods}

Subjects and surgery

Five naive subjects and the first two authors participated in the human psychophysical experiments. Two juvenile male rhesus monkeys (Ma- 


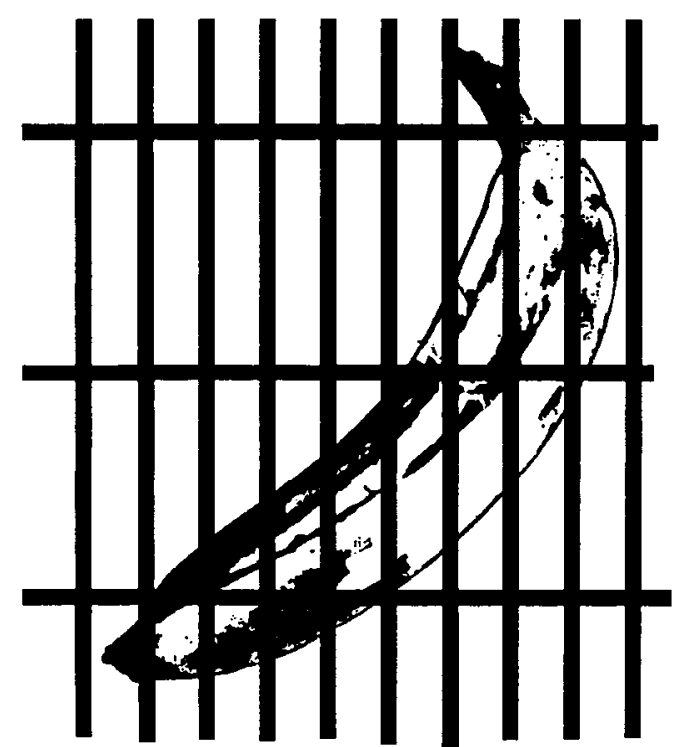

Figure 1. Illustration of the perceptual occlusion phenomenon. Although the fruit is partially obscured by the vertical and horizontal bars, one is able to recognize it as a banana. Also note that one perceives a complete banana behind bars (amodal completion), and not separate, disconnected parts beside the bars.

caca mulatta), one of which (monkey 2) was trained and tested in psychophysical experiments, served as subjects for the electrophysiological experiments. During the experiment, the monkeys were water deprived but had dry food ad libitum. Supplementary water was given to the monkeys when judged necessary. Both monkeys had a scleral search coil intulanted in one eye, using procedures similar to thuse of Judge et al. (1980), and had a stainless steel peg cemented to their skull for head fixation. The single cell recordings were done through an elliptically shaped recording chamber that was implanted laterally as described by Vogels and Orban (1994). Monkey 2 had a recording chamber implanted on either hemisphere, but all recordings were made in the one positioned over the right hemisphere. The other monkey had a recording chamber only on the left hemisphere. All surgical procedures were carried out under full anesthesia and aseptic conditions. At the end of the recording sessions, several penetrations were made in monkey 1 with stainless steel wires under ketamine anesthesia. The monkey was then sacrificed with an overdose of Nembutal and perfused with fixative. Recording sites were reconstructed by identifying the tracks of the last few penetrations in coronal brain sections $(60 \mu \mathrm{m})$ stained with cresyl violet. The other monkey is still participating in other experiments. The procedures conformed to guidelines established by NIH for the care and use of laboratory animals.

\section{Apparatus}

This is identical to that of Vogels and Orban (1990, 1994), except for changes in the stimulus generation and computer hard- and software. The monkey was seated in a primate chair with its head fixed, allowing eye movement measurements with the search coil technique. The experiment was controlled by an Intel-based 80486 PC which also sampled (rate $=200 \mathrm{~Hz}$ ) and displayed the eye movements on line. A second 486 PC sampled spikes, stimulus, and behavioral events $(1000$ $\mathrm{Hz}$ sampling rate), and displayed them on line as rasterplots and averaged peristimulus time histograms. Single cell recordings were made with glass- or parylene-coated tungsten microelectrodes held by a Narishige microdrive. The electrode signal was amplified, filtered, and single cells isolated using an amplitude window discriminator, and in later sessions, using a commercial spike discriminator system (SPS-8701 Real-Time Waveform Discriminator System, Prospect, Australia). Stimuli were generated on a BARCO CD233 monitor (frame rate $50 \mathrm{~Hz}$; P22 phosphor) using dedicated hardware developed in the Biophysics Institute (J. J. Koenderink) of Utrecht, Nederland. A black mask with a circular aperture $\left(13^{\circ}\right.$ diameter $)$ covered the monitor screen. The background luminance in the experimental room was $1 \mathrm{~cd} / \mathrm{m}^{2}$.

\section{Stimuli}

Shape stimuli. The stimuli consisted of shapes presented on a textured background, a random dot pattern with a $50 \%$ density and a dot size of 5 arc min, which was replaced on every trial. The average luminance and contrast of the background pattern was $8 \mathrm{~cd} / \mathrm{m}^{2}$ and $95 \%$, respectively. Two kinds of shape stimuli were used: black outlines (Fig. $2 A$ ) and filled shapes (Fig. 2B). Where filled shapes were used, the inside of the shape had the same luminance $\left(2 \mathrm{~cd} \mathrm{~m}^{-2}\right)$ as its outline. The outline stimuli had a thickness of 15 arc min, a contrast of $95 \%$ and the same luminance as the black dots of the background pattern $(0.5$ $\mathrm{cd} / \mathrm{m}^{-2}$ ). The areas of the various shapes were similar (approximately $10^{\circ 2}$ ). The shapes consisted of simple geometrical forms (see Fig. 5A), identical to those used by Sáry et al. (1993). They were composed of simple features such as oriented line segments so that several shapes shared the same elementary features. The shapes were presented foveally, centered on the fixation target.

Occluding patterns. The occluding pattern consisted of randomly positioned, filled squares (size $0.6^{\circ}$; Fig. $2 C$ ). This pattern was sufficiently irregular that single shape components were not completely occluded (which would have occurred when using more regular grid patterns) but orderly enough to allow quantitative characterization. The density of the occluding pattern was manipulated by changing the probability of the presence of a square at a given position. We used $50 \%$ as the standard density, which gives sufficient occlusion of the shape without impairing shape discrimination (see Results) or recognition. We used four types of these occluding patterns: moving visible (MV), static visible (SV), moving invisible (MI), and static invisible (SI). In the moving conditions, the pattern moved downward in one of the two diagonal directions with a speed of $3 \% \mathrm{sec}$. In the visible conditions, the squares were black with the same luminance as the shape outlines. In the invisible conditions (Fig. $2 D$ ), the elements of the occluder were filled with the background random dot pattern, and, thus, were not visible. In the MI condition, the background as well as the invisible occluding pattern moved at the same velocity to prevent figure-ground segregation of the occluding and background patterns. In these invisible conditions, the occluding pattern was, although present, not detectable. Thus, in the visible conditions, the occluding pattern and the nonoccluded shape elements were visible, while in the invisible conditions only the nonoccluded shape elements were detectable (this amounts to the presentation of a shape with partially deleted elements). The invisible occluding pattern was superimposed on the shape. This is different from mere masking with a random dot pattern since the nonoccluded shape elements were not masked by the random dot noise, but were presented on top of the background noise (Fig. $2 D$ ), as with the other stimulus types. The occluding pattern filled the screen completely and its position with respect to the shapes was randomized (range was 1 and $5^{\circ}$ for the moving and static occluders respectively) between trials.

\section{Stimulus sequences and behavioral tasks}

The same stimulus sequence (Fig. 3) was presented in the psychophysical and single-unit recordings. A trial was initiated by the onset of a red fixation target (size, $10 \mathrm{~min}$ arc), which was superimposed on incoherent, dynamic random noise. The latter was also present throughout the intertrial interval $(1 \mathrm{sec})$. If the monkey fixated the target, the background texture pattern was shown instead of the dynamic noise. If occlusion was involved, the occluding pattern was also presented immediately after fixation onset. The shape was presented after $700 \mathrm{msec}$ of fixation. In the single cell recordings (Fig. $3 A, B$ ), the monkey was rewarded for fixating the target until shape offset, but was not required to perform an active shape discrimination. However, in order to associate the presentation of the shape and not of the fixation target with the reward, reinforcement was delivered immediately after shape offset while the fixation spot remained on for a variable period of time (range, $700-1000 \mathrm{msec}$ ).

In the monkey psychophysical experiments (Fig. $3 \mathrm{C}$ ), two square targets were presented after shape offset, flanking the fixation target at distances of $6^{\circ}$ to the right and left. The monkey was taught to make a saccadic eye movement to the left or the right square after presentation of a shape, four of the eight shapes being associated with one side, and the remaining four with the other side. Correct responses were rewarded with a drop of apple juice.

In most of the single-cell recording sessions, the presentation time of the shape was $500 \mathrm{msec}$ (Fig. 3A). The presentation time was $300 \mathrm{msec}$ in all of the psychophysical experiments except one (see below), as well 

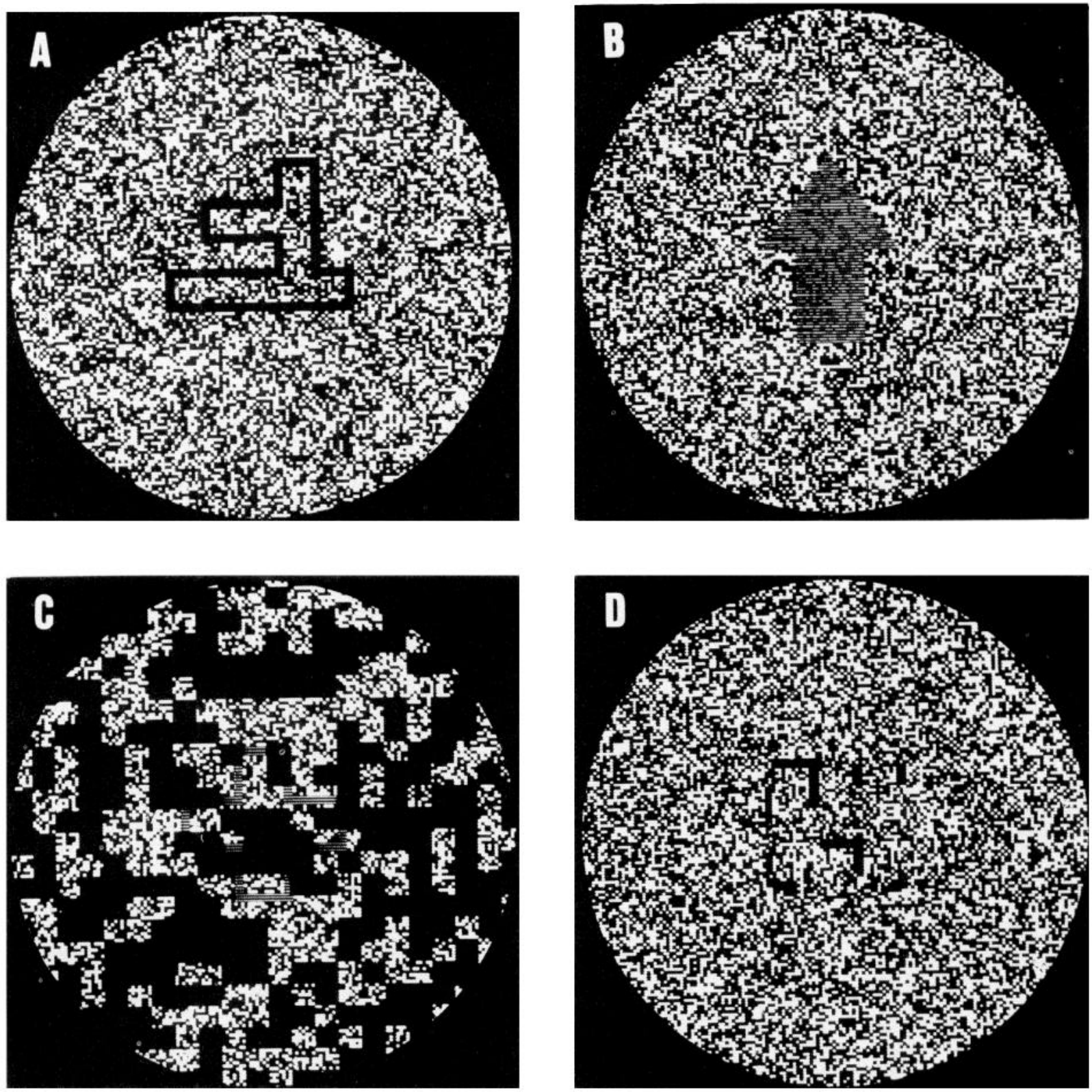

Figure 2. Photographic reproduction of some of the stimuli. A, Shape outline (labeled VI in Fig. 5) presented on the textured random dot background. B, Filled shape (labeled III in Fig. 5). C, Shape outline (labeled V in Fig. 5) and the occluder pattern (50\% density). Unlike in this figure, shape outline and occluder had the same luminance in the experiment. In the experiment, segmentation of the shape and the static occluder was made possible by the temporal onset asynchrony of occluder and shape. To help the reader, this segmentation cue is mimicked here by a luminance difference of shape and occluder. D, Shape outline (labeled IV in Fig. 5) partially occluded by an invisible occluder (50\% density).

as in some of the later recordings. In a separate experiment, we manipulated the presentation time of the stimulus, with and without the MV occlusion condition. This was done by varying the stimulus onset asynchrony between the stimulus shape and a complex pattern which served as mask (Fig. 3B). The mask was present (behind the occluder) until $500 \mathrm{msec}$ after shape onset, at which time either juice was delivered (fixation task in the recordings) or the choice squares appeared (psychophysical experiments)

In the human psychophysical experiments, the stimulus sequence was the same as that of the single-cell recording sessions, except that the fixation spot was present continuously and no feedback (i.e., reward) was given. No eye movements were measured in the human psychophysical experiments and the subjects initiated the trial by pressing a button. Each figure was associated with one of eight response buttons and the subjects had to press one of these buttons after stimulus presentation, thus indicating their response and initiating the next trial.

\section{Single-cell recording protocol and data analysis}

We searched for shape selective single cells while presenting the eight shapes without any occlusion. Once a cell was found to be responsive, we ran tests comprising 16 conditions each, one-half corresponding to the eight shapes occluded by one of the four patterns ( $50 \%$ density) and the other half consisting of presentations of the eight nonoccluded shapes. These conditions were randomly intermixed, and at least 10 trials of each condition was presented. Only those trials in which the monkey did not break fixation were taken into account. Where the density of the occluding pattern was manipulated, the 16 conditions consisted of presentations of the eight shapes with $20 \%$ and $90 \%$ of the field occluded. A somewhat different strategy was followed in studying the effect of presentation time. Firstly, the shape selectivity of the neuron was determined in the no-occlusion condition. We then ran a test in which we presented two shapes in an interleaved fashion, choosing 

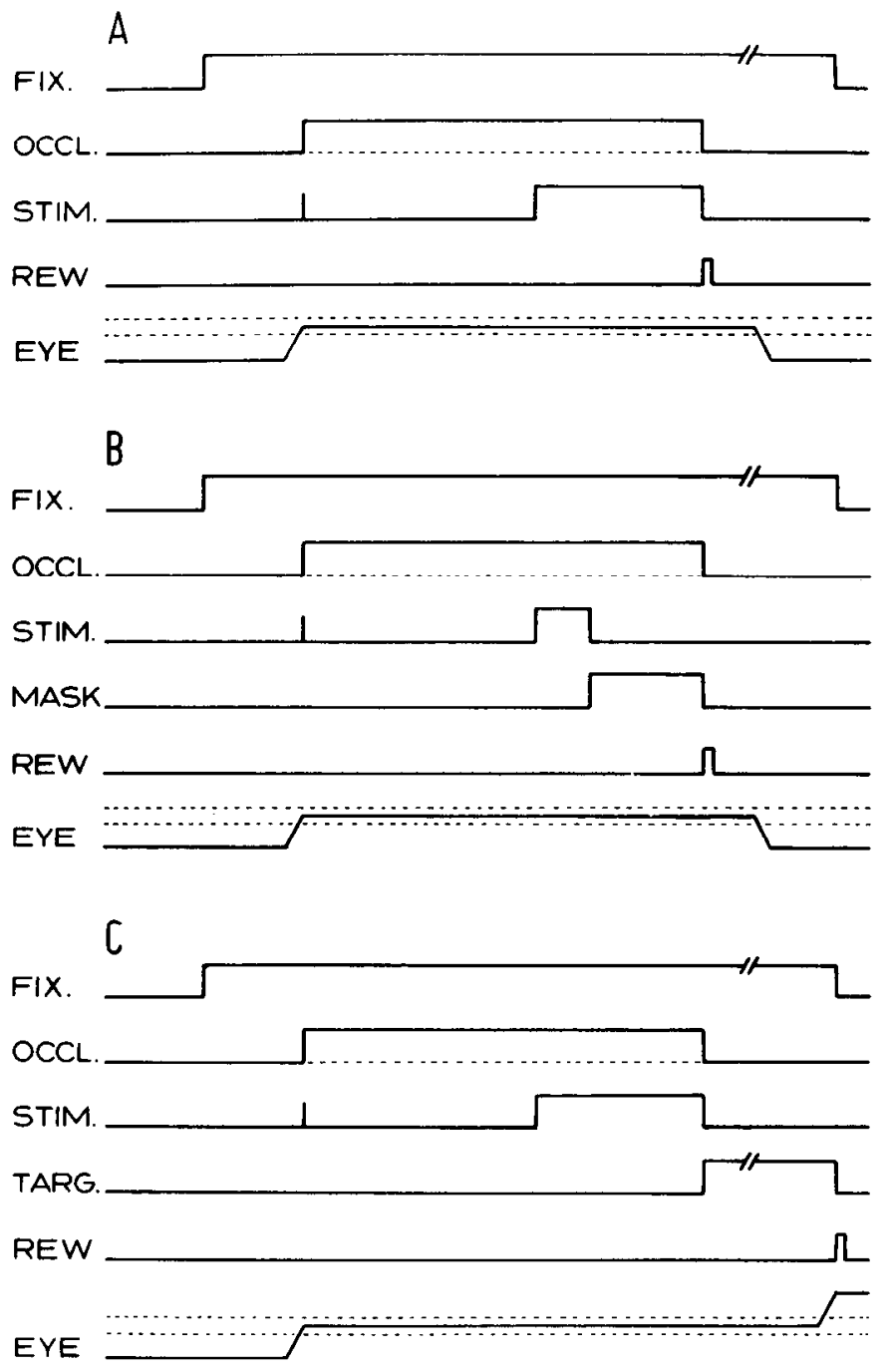

Figure 3. Stimulus sequences in the fixation and discrimination tasks. $A$ and $B$, Fixation task used in single-cell recordings. In the intertrial interval the monitor screen was filled with incoherent dynamic noise. The trial started with onset of the fixation spot $(F I X$.). When the monkey fixated (see $E Y E$ for a trace of the horizontal eye movements), the dynamic noise became stationary (vertical line in STIM.), and on 50\% of the trials the occluder pattern was presented (OCCL.). The shape was presented $700 \mathrm{msec}$ after fixation $(S T I M)$. In most experiments, the shape exposure duration was fixed $(A)$, but in the stimulus presentation time experiments $(B)$, the shape duration was randomly varied over trials (see Materials and Methods). In the latter experiments, shape offset was followed immediately by another pattern which served as mask (MASK in B). Shape $(A)$ or mask $(B)$ offset was followed by a juice reward $(R E W$.$) if the monkey had continued fixation during the trial$ $(E Y E)$. The fixation spot was extinguished at a variable time after stimulus offset in order to reduce the association of reward and fixation spot. $C$, Discrimination task used in the monkey psychophysical experiments. The same stimulus sequence as in $A$ or $B$ except that after stimulus or mask offset (not shown), two target squares were presented $(T A R G)$, to the left and right of the fixation spot. The monkey had to make a saccadic eye movement to the target associated with the shown shape. Saccades of the correct direction (left or right) $(E Y E)$ were rewarded with juice.

those eliciting the largest and smallest response in the previous test. These were presented at four different presentation times $(40,80,160$, and $320 \mathrm{msec}$ ) with and without the MV occlusion (50\% density).

Off-line spike counts were computed trialwise with a 300 or 500 msec bin that started $50 \mathrm{msec}$ after shape onset for the standard presentation time conditions. For the presentation time experiment, these
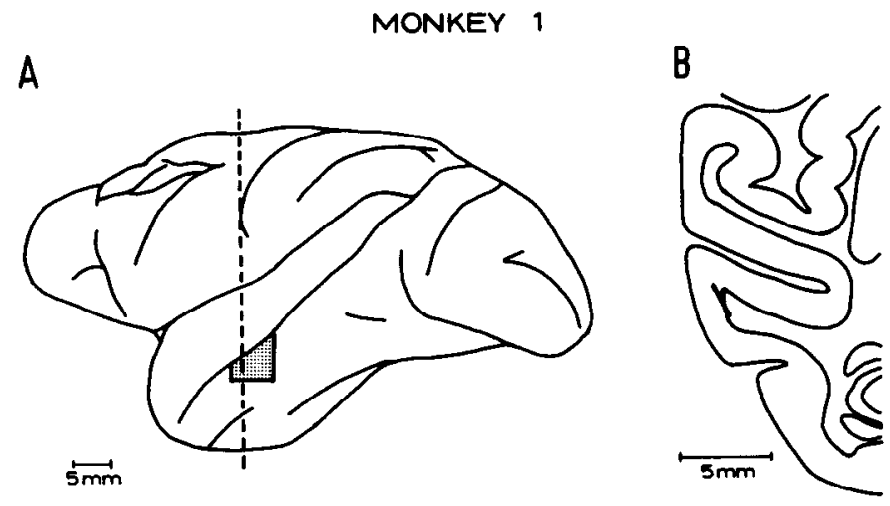

Figure 4. Recording sites in monkey 1. A, Lateral view of the left hemisphere. The range of the anterior-posterior recording positions is shown by the hatching. $B$, Coronal section at a position indicated by the vertical dashed line in $A$. The track of a penetration in the upper parts of the inferior temporal cortical convexity is indicated by the line.

time windows were adapted to the stimulus duration. Net responses were calculated by trial-wise subtraction of the activity during a fixation period of the same duration as the stimulus time window, but just preceding shape onset. We used analysis of variance (ANOVA; Kirk, 1968; also see Vogels and Orban, 1994) to test the significance of the responses to any shape and the significance of shape selectivity. Tests were classified as significant if the corresponding type $I$ error was smaller than 0.05 . The responses with and without partial occlusion were compared for each cell and for each occlusion condition by subtracting the average net response to the preferred shape in the occlusion condition from the average net response to the preferred shape in the noocclusion condition, and dividing this difference by the sum of the two responses (responsivity index).

\section{Results}

We recorded 111 shape selective neurons in the IT cortex of two monkeys (monkey 1,73 units; monkey 2, 38 units). Histological analysis of the brain of monkey 1 showed that the recordings were made in the central part of area TE, excluding area TEO (Fig. 4). The units were recorded in the lateral part of the lower bank of the superior temporal sulcus (STS) and the dorsal part of the inferior temporal cortical convexity. Stereotaxic measurement of the anterior/posterior position of the recording chamber of monkey 2 indicated that his chamber was positioned more posterior ( $11 \mathrm{~mm}$ anterior to the external auditory meatus) than that of monkey 1, and may have included anterior parts of area TEO. Thus, together the two monkeys provide neuronal data from a wide range of anterior/posterior positions in IT.

We will present the results of the single-cell recordings in three main sections. In each section we will also report the results of the psychophysical experiments, demonstrating that the monkey shows, at least qualitatively, the same shape discrimination abilities as humans under the occlusion conditions used in these single-cell recordings. In the first section, we will determine whether the IT neurons preserve their shape selectivity if these shapes are partially occluded and the occluder is visible. We will then compare the effect of presentation time on shape selectivity for nonoccluded and partially occluded shapes. In all these experiments we have used the shape outlines as stimuli. In the third section, we will examine whether IT units respond in a different manner to the elements of the same shape when these are perceived as either part of a completed figure (amodal completion) or as separated elements. This will be tested by comparing the responses under the visible (amodal completion) and invisible (no amodal completion) occlusion conditions, using both the outline and filled shapes. 


\section{Discrimination of partially occluded shape outlines}

Psychophysical observations

Humans discriminated the eight shape outlines perfectly (100\% correct; 100 trials per subject) when they were partially occluded by MV and SV patterns with a density of $50 \%$ or less. Monkey 2 was also tested for the ability to discriminate the eight shapes when these were partially occluded by the MV pattern. First, the monkey was extensively trained to discriminate the eight shapes without occlusion. Then, trials with gradually increasing occlusion density were intermingled with nonocclusion trials. The monkey performed with $83 \%$ correct in the first 160 occlusion trials, indicating transfer of the shape discrimination from the no-occlusion condition to the occlusion conditions. This suggests that he did not learn independently, which occluded patterns were associated with which response, but recognized the occluded shapes as identical to the nonoccluded shapes. Discrimination performance was formally measured under no-occlusion, $50 \%$ and $90 \%$ occlusion density conditions of the MV pattern. As with the human subjects, the monkey was able to discriminate the eight shapes when they were partially occluded with a density of $50 \%$ ( $91 \%$ correct; SE, $2 \% ; N=2640$ trials). However, the response latency was significantly longer (MannWhitney $U$ test; $U=0 ; p<0.01$ ) in the $50 \%$ occlusion condition [mean, $531 \mathrm{msec}(\mathrm{SE}, 6 \mathrm{msec})]$ compared to the no-occlusion condition [mean, $457 \mathrm{msec}$ ( $\mathrm{SE}, 3 \mathrm{msec}$ )]. Occlusion with the $90 \%$ density pattern, rendering the shapes nearly invisible, yielded a discrimination performance at chance level $(52 \%$; SE, $4 \% ; N=320$ trials).

These results demonstrate that the monkey, like the human subject, is able to discriminate shapes under the $50 \% \mathrm{MV}$ occlusion condition. We used this occlusion condition as a standard for determining the effect of partial occlusion on the responsivity and selectivity of IT neurons.

\section{Single cell recording}

Effect of partial occlusion on responsiveness and shape selectivity: basic observations with $M V$ pattern. We recorded the responses of 80 shape selective neurons under conditions of noocclusion and occlusion with the MV pattern (density $50 \%$ ). For 50 of these, we compared the selectivity using the eight shapes, while for 30 others we compared only the responses to two shapes, being the preferred and nonpreferred shapes from the no-occlusion condition. The latter data were derived from the presentation time experiment (see next section).

An example of a unit tested with the eight shapes with and without MV occlusion is shown in Figure 5. This unit showed shape selectivity under the no-occlusion condition (1st and 3th row in Fig. 5A), responding most strongly to the inverted $\mathrm{F}$ and the star shapes. When the shapes were partially occluded (2nd and 4th row of Fig. $5 \mathrm{~A}$ ), the unit still responded to these shapes, and, in fact, retained its overall shape selectivity. The net response strength of this particular unit was similar in the noocclusion and MV conditions (see Fig. $5 B$ ).

In general, the units responded less to a given shape when it was partially occluded compared to the no-occlusion condition. Figure 6 shows the distribution of the responsivity index (see Materials and Methods), a positive value indicating a larger response in the no-occlusion condition. The median responsivity index was 0.26 (1st quartile, $0.09 ; 3$ th quartile, $0.43 ; N=80$ ), indicating that overall, the response strength with partial occlusion (50\% density $\mathrm{MV}$ ) was one-half of the response strength
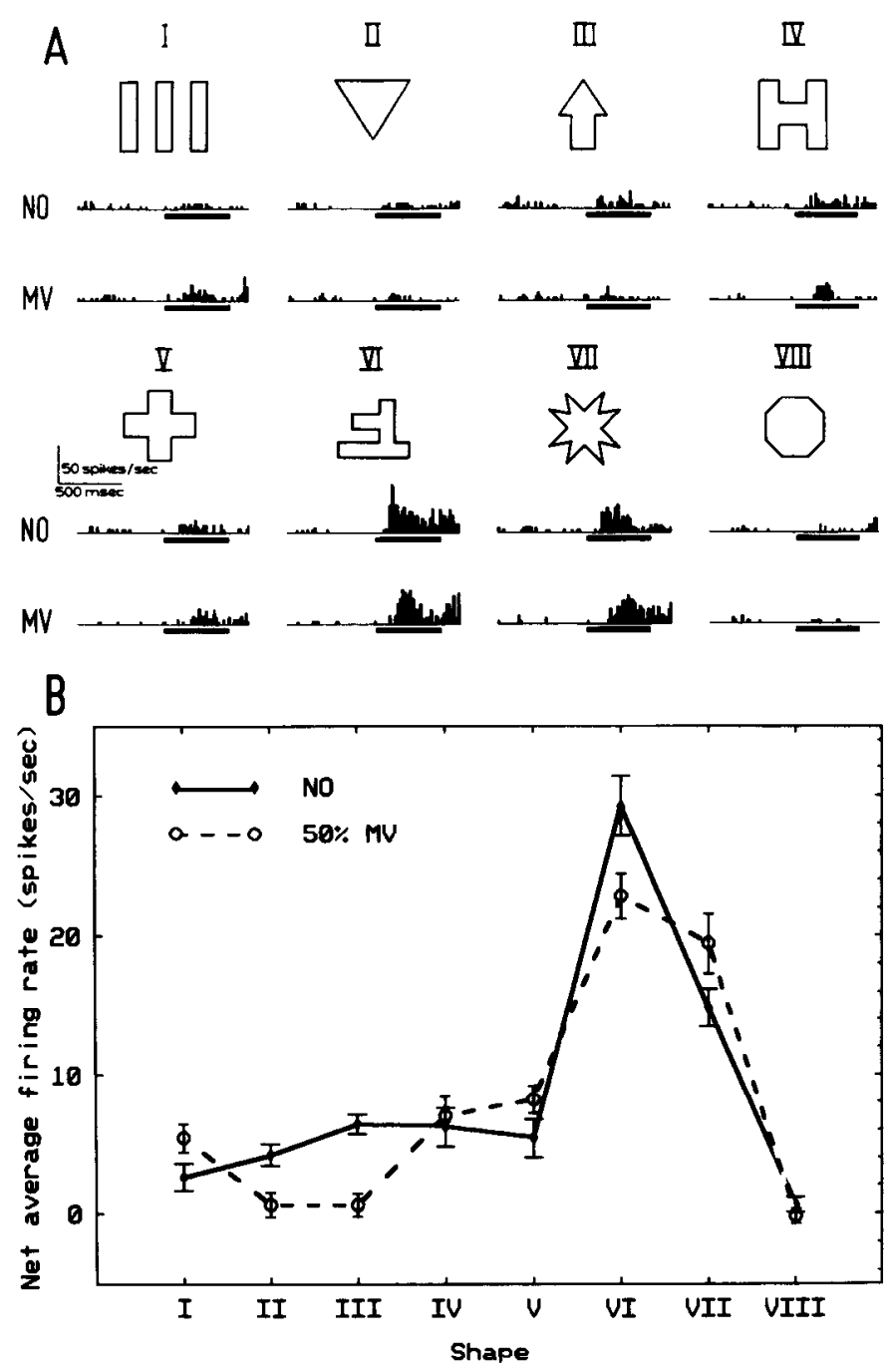

Figure 5. Shape selectivity of an inferior temporal single unit (monkey 2) with and without occlusion with the standard 50\% moving visible occluder. A, Peristimulus time histograms of the spikes for each of the eight shape outlines, shown above the histograms, in the no-occlusion ( 1 st and 3th row) and occlusion condition (2nd and 4th row). Presentation of the shapes are indicated by the horizontal bars. $B$, Response strength of the same neuron for the eight shapes with (solid line) and without (stippled line) partial occlusion. Each shape is indicated by a roman numeral as shown in $A$. Error bars represent standard errors of the mean.

with no occlusion. This smaller response in the occlusion condition also tended to be more variable [median normalized response variance (Vogels and Orban, 1991) 1.2] than without occlusion (median normalized response variance, 1.1), although the increase in variability was small and not statistically significant (Wilcoxon matched pair test, $T=339$; NS). Despite the lower level of responsivity, it was found that $79 \%$ of the units (empty blocks in Fig. 6) still responded significantly to the shapes that were partially occluded by the $50 \%$ density MV pattern. The average response latency was $108 \mathrm{msec}$ and 158 msec in the no-occlusion, and MV condition, respectively, a difference that is statistically significant (Wilcoxon matched pair test; $T=14 ; p<0.0001 ; N=54$; Table 1 ).

The unit shown in Figure 5 showed the same shape preference with or without partial occlusion. This was a general finding as 


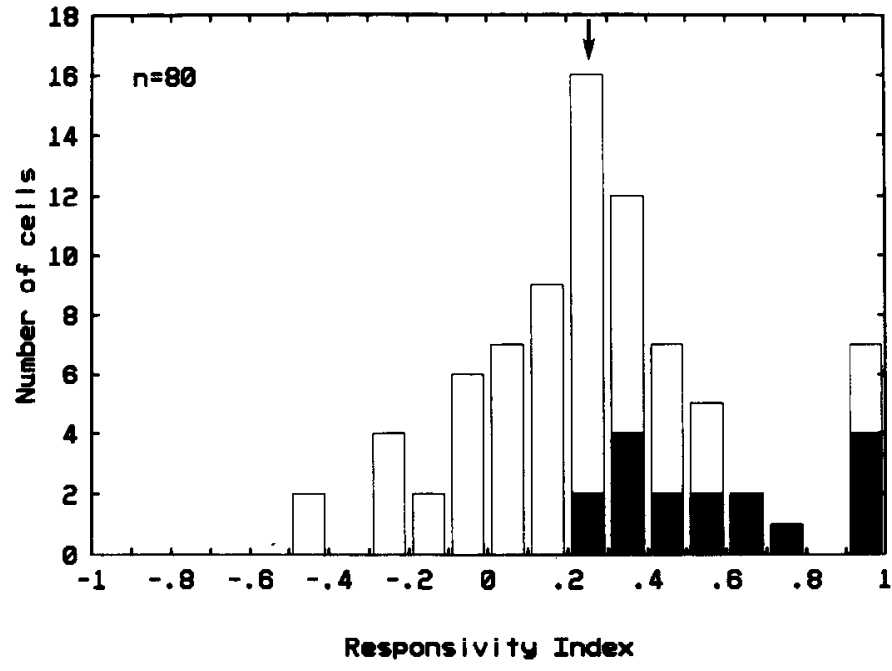

Figure 6. Distribution of the responsivity index (see Materials and Methods) for 80 units tested with and without occlusion by the $50 \%$ moving visible occluder. Positive values represent units with a larger response in the no-occlusion compared to the occlusion condition. Units that did not respond when the shape was partially occluded are indicated by the filled bars. The arrow indicates the median of the distribution.

demonstrated by the following analysis of the 50 shape selective units tested with the eight shapes. For each unit and condition we ranked the eight shapes according to their net response in the no-occlusion condition. For example, for the unit of Figure 5 , the inverted $F$ shape receives rank 1 , the star rank 2 , and so on. Then we calculated the average net response for the 50 units in the no-occlusion and occlusion condition as a function of shape rank. If shape preferences in the occlusion and no-occlusion conditions were completely independent, then there would be no relationship between the average occlusion condition response and shape rank, since this ranking is based solely upon the no-occlusion condition. However, as shown in Figure $7 \mathrm{~A}$, the average net response in the occlusion condition decrcases significantly with increasing shape rank, demonstrating that the shape preference is similar with or without partial occlusion with the MV pattern.

The curve relating net response to shape rank is flatter in the occlusion compared to the no-occlusion condition. In order to determine whether this is merely a consequence of the lower responses seen in the occlusion condition or constitutes a genuine difference in shape selectivity between the two conditions, we normalized, independently, the occlusion and no-occlusion responses for each shape. This normalization nullifies absolute differences in net response between the occlusion and no-occlusion condition. These normalized responses were then averaged for each shape rank (see above). Only those units $(N=45)$ that were responsive both with and without occlusion and that were tested with all eight shapes were taken into account. As shown in Figure $7 B$, the normalization considerably reduces the difference between the no-occlusion and occlusion condition. Nonetheless, the decrease in the average normalized response with increasing shape rank is still less in the 50\% MV occlusion compared to the control condition, indicating that the occlusion affected, albeit weakly, the shape selectivity. However, the units are clearly able to respond differentially to the shapes in the partial occlusion condition: the average response to the preferred shape is about three times the response to the nonpreferred shape
Table 1. Neuronal latency differences

\begin{tabular}{llll} 
& V-NO & I-NO & V-I \\
\hline M/OUTL & $* 50 \pm 4$ & $1 \pm 10$ & $* 42 \pm 11$ \\
& $(n=54)$ & $(n=14)$ & $(n=10)$ \\
S/OUTL & $* 25 \pm 7$ & $* 13 \pm 5$ & $11 \pm 9$ \\
& $(n=15)$ & $(n=15)$ & $(n=14)$ \\
S/FILL & $* 20 \pm 5$ & $* 21 \pm 6$ & $-4 \pm 7$ \\
& $(n=16)$ & $(n=12)$ & $(n=11)$
\end{tabular}

Differences in response latencies (average \pm SE) between conditions as indicated. V, visible occluder; I, invisible occluder; NO, without occlusion; $M$ moving occluder; S, static occluder; OUTL, shape outlines; FILL, filled shapes. $n$, number of observations.

* Statistically significant difference (Wilcoxon matched pair test, $p<0.05$ ).

after partial occlusion. These results (Fig. 7) imply that after partial occlusion, despite the reduction in average net response (Figs. 6, 7A), the units remain sufficiently selective, at least as a population, to signal a particular shape.

\section{Effect of the occluding pattern density}

For 14 cells we tested the effect of the density of the MV occluder pattern. Figure $8 \mathrm{~A}$ shows the average net response as a function of the shape rank as determined by the responses to the nonoccluded shapes. The response strength decreases monotonically with increasing occluder density. This is confirmed by examining the median Responsivity Indices, which were 0.09 , 0.26 , and 0.66 for the $20 \%, 50 \%$, and $90 \%$ densities, respectively. Although most cells (12/14) were still significantly responsive in the $90 \%$ occlusion condition, these responses were small and the curve relating average net response to shape rank was nearly flat. The shape selectivity of those cells still responding (weakly) in the $90 \%$ condition differed from that in the other conditions as apparent in Figure $8 B$. It can be seen that the average normalized response did not decrease monotonically as a function of shape rank in this condition, in contrast to the other two occlusion conditions. In fact, the only differences to be found in the responses of the $90 \%$ condition were between the preferred shape (rank 1) and the remaining shapes, but even this was not statistically significant [ANOVA, $F(7,77)=1.59$; NS] Although the response strength was less in the $50 \%$ than in the $20 \%$ density condition (Fig. $8 A$ ), there was little difference in the shape selectivity for these two conditions as shown by the similarity of the curves relating the average normalized response in these two conditions to the shape rank (Fig. 8B).

Behaviorally, shape discrimination, as well as recognition, is unaffected by occlusion for the 20 and $50 \%$ density patterns, while shape discrimination is strongly impaired at $90 \%$ density. In the 20 and $50 \%$ occlusion condition, the differences in neural responses among the shapes seem to be large enough to be discriminable, while the responses may be too weak in the $90 \%$ condition to discriminate among shapes. Thus, it appears that both shape selectivity and response strength must be sufficiently strong if reliable shape discrimination is to be achieved.

\section{Partial occlusion with the SV pattern}

Similar results were obtained using the SV in place of the MV occluding pattern. Fifteen of the 16 units tested with the SV occluder remained significantly responsive when occluded with that pattern. The median responsivity index was 0.17 (quartiles, $0.04-0.24 ; N=16$ ), a value not significantly different (Mann- 

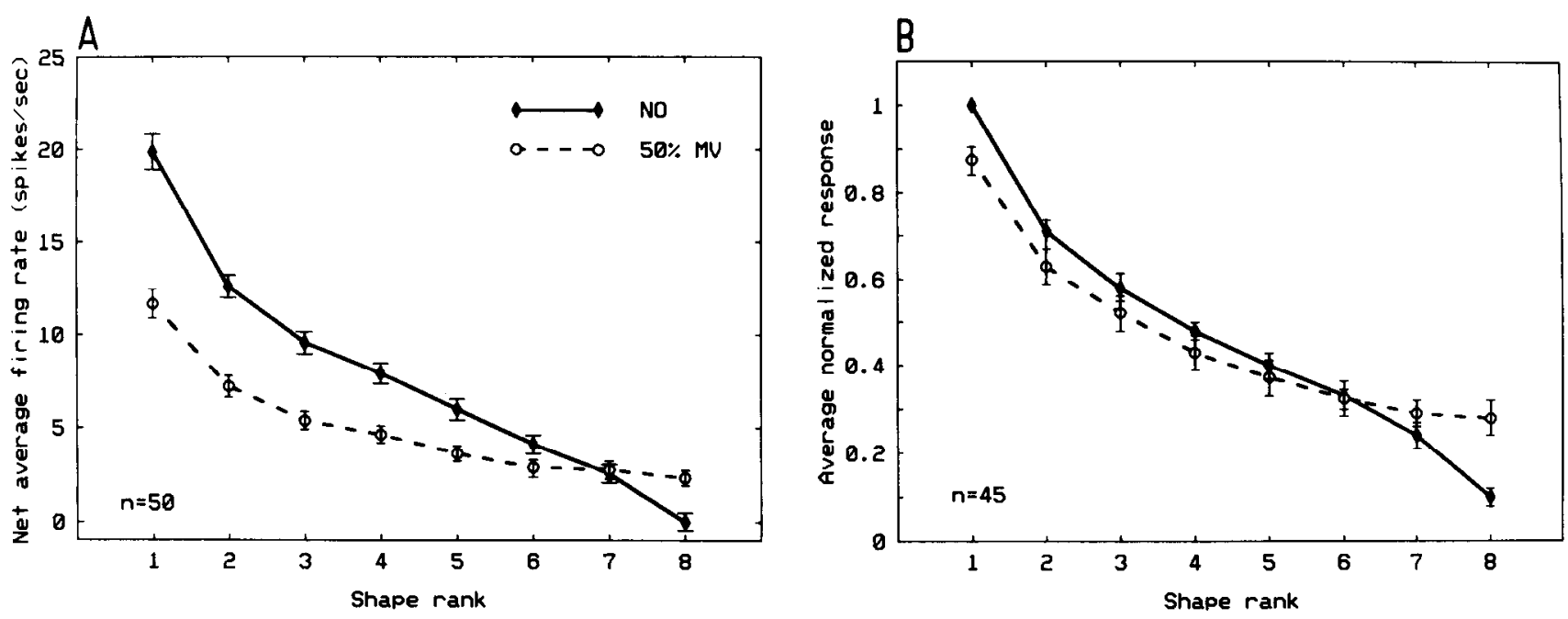

Figure 7. Selectivity of inferior temporal neurons for partially occluded shape outlines: the standard $50 \%$ moving visible condition. A, Average response strength as a function of the shape rank with (stippled line) and without partial occlusion (full line). The shape rank was determined using the responses in the no-occlusion condition only. Standard errors of the mean are indicated. $B$, Averaged normalized responses of those cells also responsive in the occlusion condition as a function of shape rank. The normalization was done for each unit and occlusion condition separately, canceling differences in response strength between the no-occlusion and occlusion condition. Same conventions as in $A$.

Whitney $U$ test, $U=496$; NS) from that obtained with the MV pattern $(0.26)$, indicating that, as in the MV condition, the average response was smaller with than without the SV occlusion. However, the average difference in latency between the SV and no-occlusion condition was $25 \mathrm{msec}$, half of the latency difference for the MV and no-occlusion conditions.

As for the MV occlusion pattern, the average shape preference was the same with or without partial occlusion by the SV pattern (Fig. 9): the average normalized response in the SV condition decreased monotonically with the shape rank as determined in the no-occlusion condition. However, the latter curve was flatter in the occlusion condition, indicating that the shape selectivity was affected. Nonetheless, the average response to the preferred shape was still three times larger than the nonpreferred shape in the SV occlusion condition. Note the similarity of the curves representing the two types of occlusion in Figure 9, indicating that moving and static occlusion have a similar, weak effect on the shape selectivity. These results show that the obscrvations made using the MV pattern are not due simply to the motion of the occluding pattern, since similar results are obtained when the occluding pattern is static (SV occlusion).

\section{Shape discrimination at short stimulus exposures under partial occlusion}

\section{Psychophysical observations}

We found that at short stimulus exposures, shape discrimination was strongly impaired under partial occlusion conditions. This provided a means to compare physiological and behavioral results. With this in mind, we compared the effect of stimulus
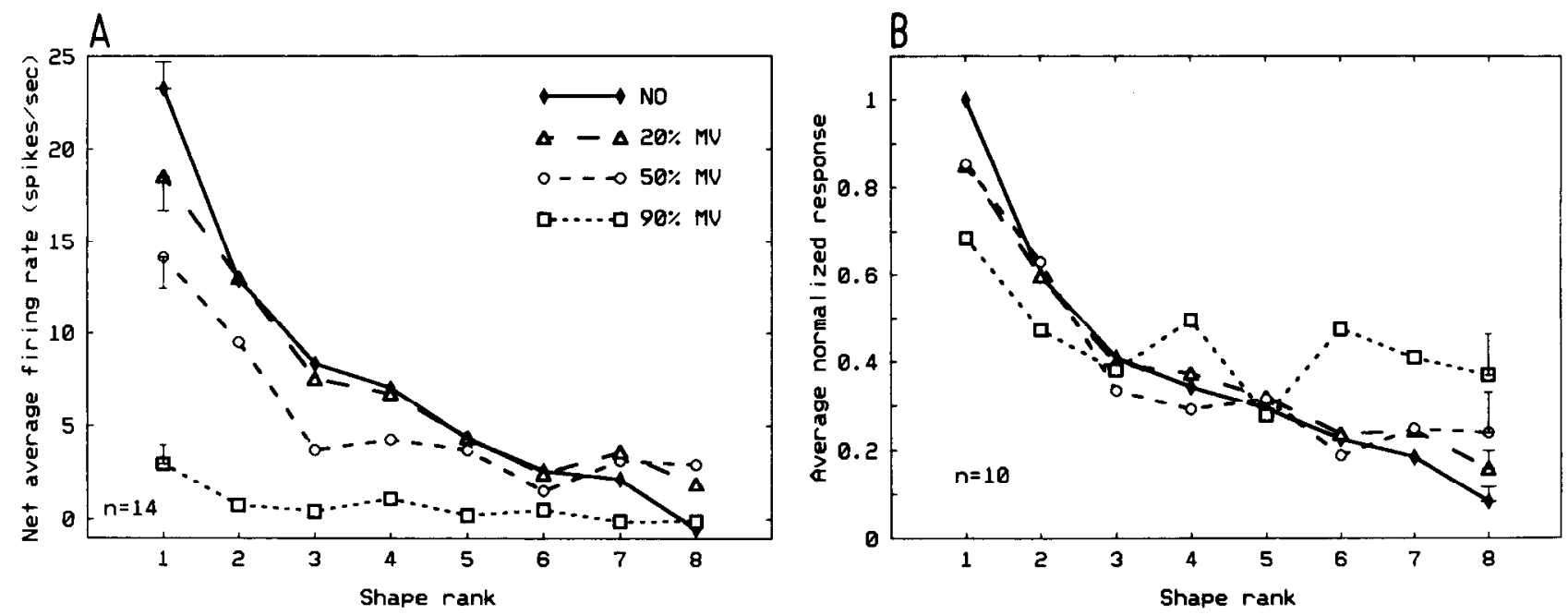

Figure 8. Effect of occluder density on shape selectivity of inferior temporal neurons. A, Average response strength as a function of the shape outline rank at four different degrees of partial occlusion (moving visible occluder). The shape rank was determined using the responses in noocclusion condition. Standard errors of the mean are indicated. B, Averaged normalized response of those cells responsive in the three occlusion density conditions as a function of shape rank. The normalization was carried out for each unit and density condition separately. For sake of clarity, only a few representative standard errors of the mean are shown. 


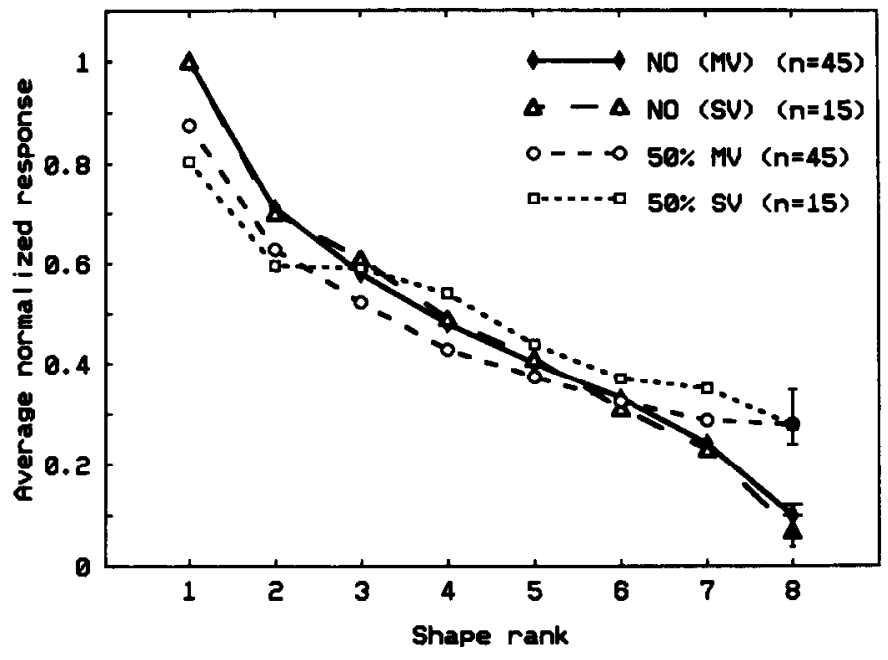

Figure 9. Selectivity of inferior temporal neurons for partially occluded shape outlines: the static and moving visible condition (50\% density) compared. Averaged normalized response of those units responding in the occlusion conditions as a function of shape rank. The shape rank was determined using the responses in the no-occlusion condition and the normalization was done for each unit and occlusion condition separately. Representative standard errors of the mean are indicated. Note the similarity of the curves of the two no-occlusion conditions that were paired with the static $(S V)$ and moving occlusion $(M V)$ condition, respectively.

duration on shape discrimination and selectivity in the MV and no-occlusion conditions.

Figure 10 shows the results of the psychophysical experiment. The chance level in the human experiment (Fig. 10A) was $12.5 \%$, since there were eight response alternatives, but $50 \%$ for the monkey with only two alternatives (Fig. 10B). Both species showed qualitatively the same effect with regard to shape duration: at long stimulus duration shape discrimination in the occlusion and the no-occlusion condition are identical, while at shorter presentation times shape discrimination in the occlusion condition becomes progressively impaired compared to the noocclusion condition. Thus, discrimination of partially occluded shapes requires longer stimulus presentation than discrimination of non-occluded shapes.

\section{Single cell recording}

We varied the exposure duration using the same backward masking procedure as in the psychophysical experiments in 39 units. As explained in the Materials and Methods section, neuronal responses to the preferred (rank 1) and nonpreferred (rank 8) shape were measured at four stimulus duration with and without occlusion. The results from one unit are shown in Figure 11. This unit also responded to the onset of the occluding pattern, a phenomenon observed in $31 \%$ of the units. The response to the preferred shape declined with decreasing presentation time. However, the effect of exposure duration was much stronger in the occlusion than in the no-occlusion condition. In the no-occlusion condition, there was still a shape response at $40 \mathrm{msec}$ exposure duration, but not in the occlusion condition. The effect of duration on the shape selectivity can be clearly shown by subtracting the nonpreferred from the preferred response, for each occlusion and duration condition. This subtraction also eliminates the responses to the masking pattern presented immediately after the shape. Figure 11 illustrates how that unit was able to differentiate the two shapes at all durations in the noocclusion condition, but failed to do so with occlusion at 40 msec.

The absence of shape selectivity at short stimulus durations in the occlusion condition was a general property of IT units, as illustrated in Figure 12. For this figure, we first normalized the responses in the 16 conditions for each unit, and then computed the average of these normalized histograms for the 39 neurons. The resulting histograms, shown in Figure 12, indicate that the average shape response declines more strongly with decreasing stimulus duration in the occlusion than in the no-occlusion con-
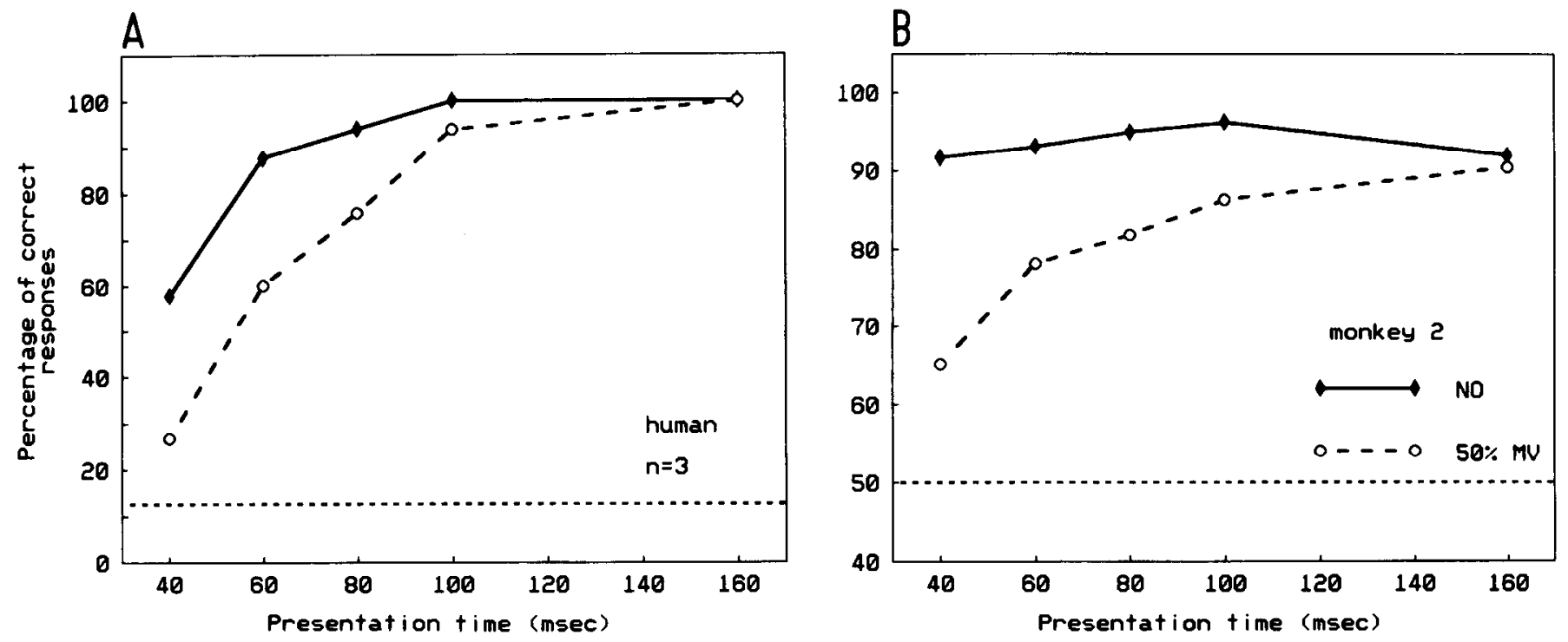

Figure 10. Effect of shape duration on the discrimination of partially occluded shapes (50\% moving visible occluder). A, Average discrimination performance of three human subjects (two authors and one naive subject) as a function of shape duration in the no-occlusion (NO) and occlusion ( $50 \% \mathrm{MV}$ ) condition. Since there were eight response alternatives, corresponding to each of the eight shape outlines, the chance level was $12.5 \%$ (horizontal stippled line). B. Discrimination performance of monkey 2 as a function of shape duration with and without occlusion. The chance level was $50 \%$ (two response alternatives) in the monkey experiments. 


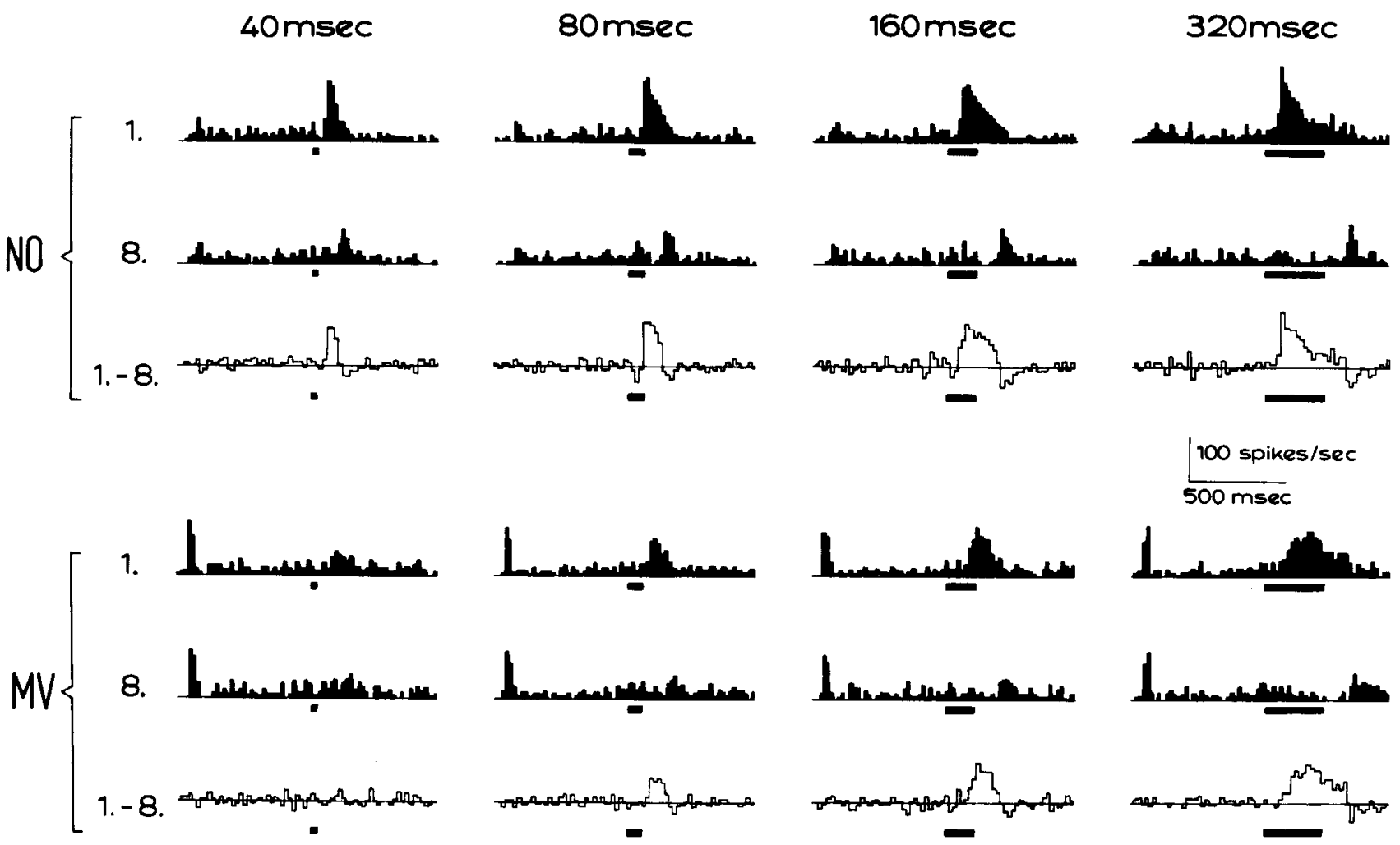

Figure 11. Effect of shape duration on the selectivity for partially occluded shapes: example one neuron in monkey 1 is shown as an example. Peristimulus time histograms of the responses in the no-occlusion (top) and 50\% moving visible occlusion condition (bottom). Each column corresponds to the exposure duration listed above the upper histograms. The first two rows of a condition are the responses to the preferred (rank 1) and nonpreferred (rank 8) shape. The horizontal bar below each histogram indicates the shape presentation. The shape was immediately followed by a mask, to which the neuron also responded (e.g., late activity in the second row of the no-occlusion condition). The third row of each condition represents the difference of the responses to the preferred and nonpreferred shape, i.e., a subtraction of row 1 and row 8 . This subtraction eliminates the responses to the mask and represents selective shape responses only. This neluron also responded to the onset of the occluder pattern, as attested by the transient responses at the beginning of the histograms of the occlusion condition.

dition, so that the shape selectivity was lower in the MV occlusion than in the no-occlusion condition at the shorter presentation times. This can be demonstrated by subtracting the normalized histograms of the nonpreferred (rank 8 ) from those of the preferred shape (rank 1). The response difference between the two shapes (see 3th and 6th row of Fig. 12) was statistically significant in all except the $40 \mathrm{msec}$ occlusion condition (ANO$\mathrm{VA} ; p>0.05$ ), in which no selectivity was present.

These data indicate that selective responses to partly occluded shapes require longer stimulus durations than nonoccluded shapes. This result fits the psychophysical observations reported above, especially those of the monkey, suggesting that the shape selective activity of these units, indeed, underlies shape discrimination.

Testing amodal completion: comparison of visible and invisible occlusion patterns

\section{Psychophysical observations}

Partially occluded shapes are not only discriminable, but are also perceived as single, complete forms. However, in order to obtain this "amodal" completion the occluding pattern must be visible to the observer (Michotte et al., 1964; Bregman, 1981). The reader can demonstrate this for himself using Figure 13: if one covers the deleted portion of the triangle of Figure $13 A$ with a pencil or one's finger, for example, one perceives a complete triangle, a part of which is occluded by the object on the draw- ing. However, removing the occluding object will change this precept into that of two disconnected forms (a demonstration originally presented by Michotte et al., 1964). This amodal completion phenomenon and its link to occluder visibility is even stronger using filled figures in place of outlines, as the reader can also demonstrate for himself using Figure $13 B$.

Shape outlines from which parts have been deleted (i.e., the invisible occlusion conditions) can still be discriminated, despite the absence of anodal completion. In fact, formal testing in three human subjects showed that the eight shapes could be discriminated with $100 \%$ correct in both the static and moving invisible conditions. The monkey, which was tested only for the moving conditions, had a discrimination performance of $92 \%$ correct (SE $2 \% ; N=800$ trials) for the eight shapes occluded by MI pattern, very similar to the $91 \%$ correct score obtained in the MV condition (see above). Also, the response latencies of the monkey were similar in the MV [531 msec (SE, 5.6)] and MI [530 msec (SE, 1)] occlusion conditions.

The discrimination of filled shapes occluded by the static $50 \%$ density pattern was tested in humans but not in the monkey. Their performance was significantly impaired when the filled shapes were partly occluded by an invisible pattern (mean, 88 $\%$ correct; 100 trials per subject) than when occluded by the visible occluder (mean, 99\% correct; Wilcoxon matched pair test; $T=0 ; p<0.02$; seven subjects). In fact, those subjects ( $N$ $=3$ ) who were unfamiliar with the shapes and who were tested 


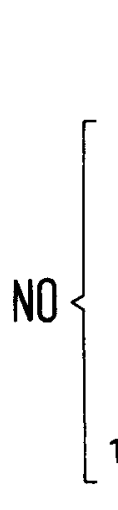

$40 \mathrm{msec}$

1.

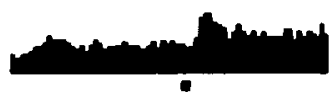

8.

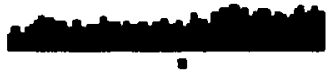

$1 .-8$.

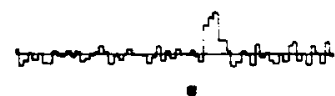

1.

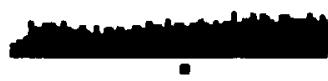

8.

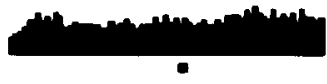

1.- 8

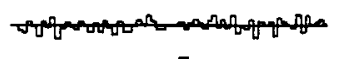

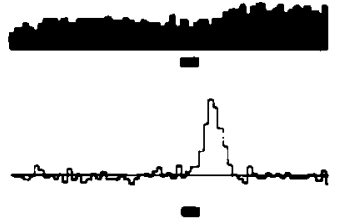

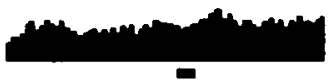

$80 \mathrm{msec}$
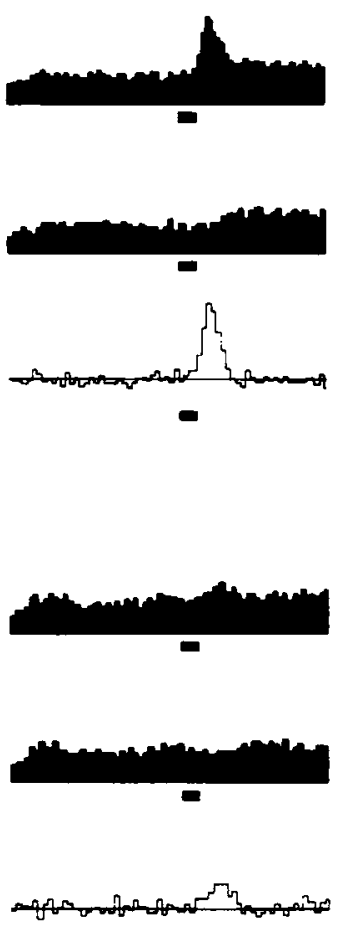

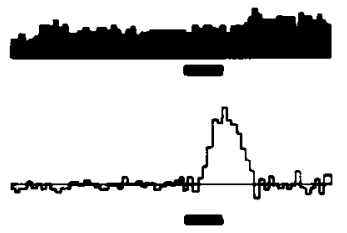

$160 \mathrm{msec}$

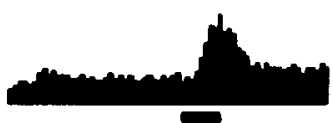

$500 \mathrm{msec}$
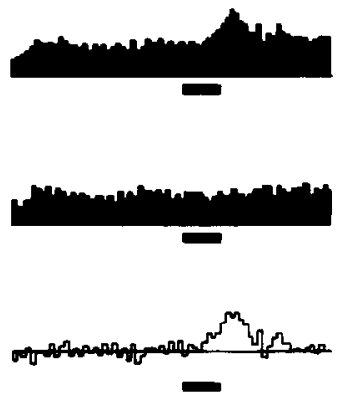
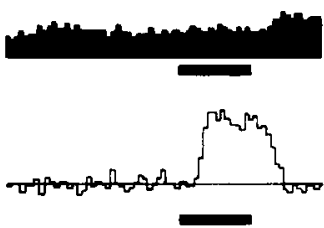

$50 \%$

$320 \mathrm{msec}$
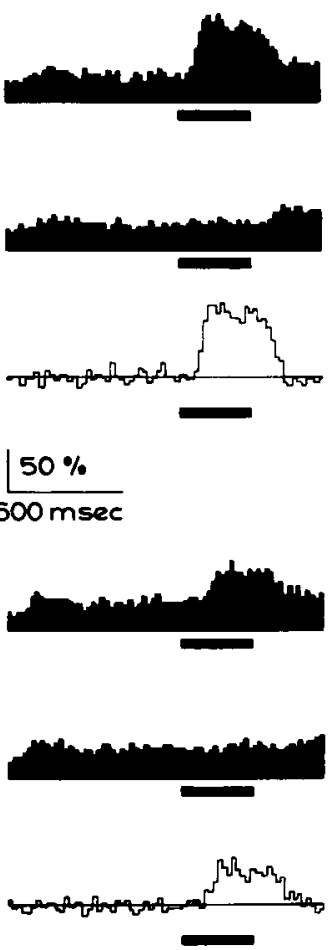

Figure 12. Effect of shape duration on the selectivity for partially occluded shapes: average activity of all cells tested. The conventions are the same as in Figure 11, except that the histograms represent the pooled normalized activity of all 39 neurons tested. For cach unit the responses werc normalized with respect to its maximum activity. The normalized histograms were then pooled across neurons.

A

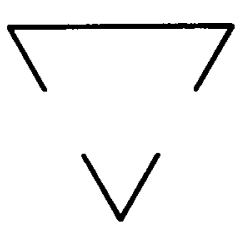

B
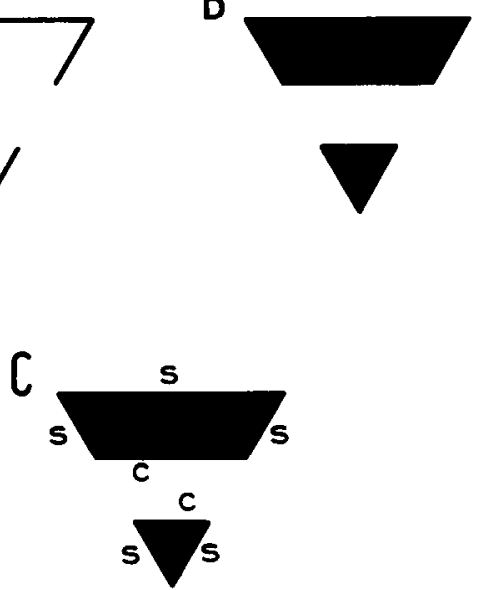

Figure 13. Demonstration of the role of occluder visibility for amodal completion (after Michotte et al., 1964). $A$ and $B$, The reader should lay his finger or a pencil horizontally over the shapes so that the horizontal gap is no longer visible. Instead of perceiving two separate parts of the shape, one will have the impression of one completed shape (a triangle) behind the occluding finger or pencil. $C$, A filled shape, parts of which are deleted by an invisible occluder, has two kinds of boundaries: those belonging to the shape itself $(s)$ and those common to the occluder and the shape $(c)$. The latter boundaries close the shape part and, thus, lead to other shapes, which can be different from the one occluded (e.g., the upper part). The presence of the (visible) occluder leads to identification of the boundaries as belonging to the shape or occluder, and thus, segmentation of shape borders and occluding pattern. with SI before being tested with the SV occluder obtained only $78 \%$ correct in the SI occlusion condition. Discrimination performance for the invisible condition was much better $(94 \% ; N$ $=4$ ) when the shapes had already been used in the visible occlusion condition, i.e., when they were familiar to the subjects. The latter observations show that the difficulty in the SI condition is more the result of an impairment of the ability to recognize novel shapes than a direct effect upon shape discrimination, and also implies that shape discrimination is possible for filled shapes (when it is known in advance which shapes to expect).

\section{Single cell recordings}

We have demonstrated above that the single IT units remain selective for shape outlines under a variety of partial occlusion condition. However, it is possible that these cells are merely responding to the nonoccluded shape components and do not carry a representation of a completed shape, i.e., the physiological counterpart of the perceptual phenomena of amodal completion. If the cells require completed shapes instead of segmented parts, then one would expect the shape selectivity with or without visible occlusion (amodal completion) to be similar, while the selectivity for shapes that are occluded by the invisible occluding pattern (no amodal completion) would differ from the no-occlusion and visible occlusion condition. If the units' selectivity is based on the presence of particular pieces of the incom- 

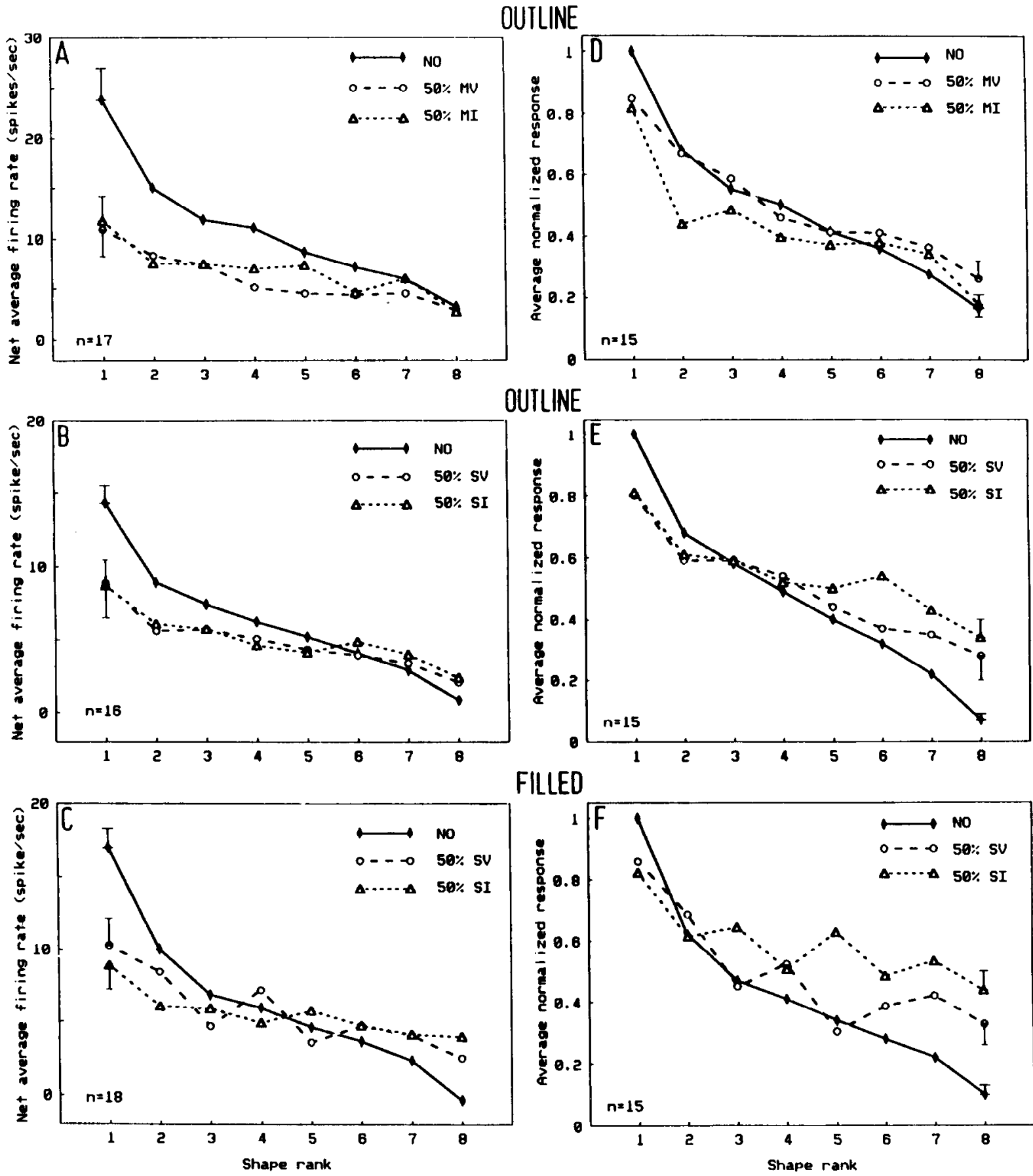

\section{FILLED}

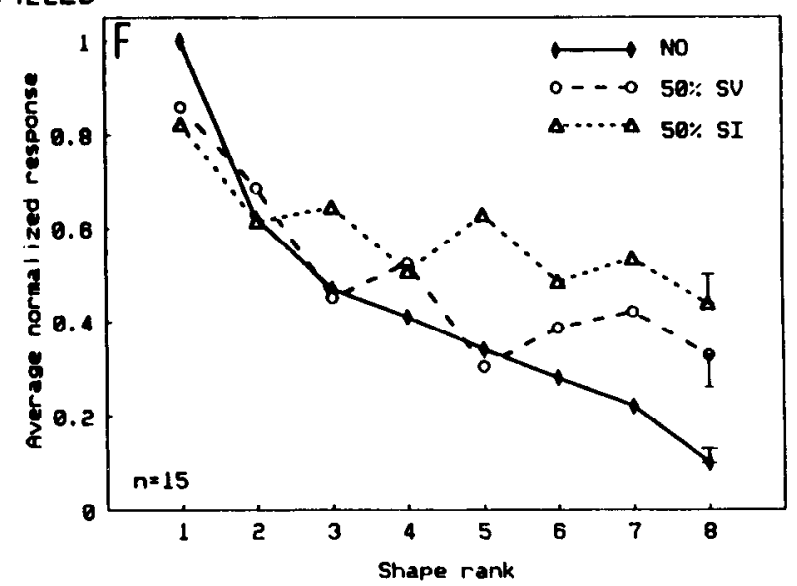

Figure 14. Effect of occluder visibility on shape selectivity. $A$ and $D$, Moving occluder (50\% density) and shape outlines. $B$ and $E$, Static occluder (50\% density) and shape outlines. $C$ and $F$, Static occluder (50\% density) and filled shapes. On the left $(A-C)$ is shown the average response of all neurons tested as a function of shape rank; on the right $(D-F)$, the average normalized response of those neurons that responded in the visible occlusion condition, is plotted as a function of shape rank. In each case, the shape rank was determined using the responses in the no-occlusion condition. $M V$, moving visible; $M I$, moving invisible; $S V$, static visible; $S I$, static invisible. All other conventions as in Figure 7.

plete outline, then shape preference would be similar in the visible and invisible occluding conditions.

Shape outlines. We compared the responses to the eight shapes, when these were nonoccluded, occluded by the MV, or occluded by the MI pattern, for 17 neurons. An additional 16 units were tested with the SV and SI occluding patterns. The average net responses for the moving and static conditions are shown in Figure 14, $A$ and $B$, respectively. These responses are expressed as a function of the figure rank, as determined by the responses in the no-occlusion condition. The curves for the invisible and visible occluder conditions are similar for either moving or static patterns. For all four conditions, the average response is less with occlusion, regardless of the visibility of the occluder. This observation also implies that the diminished average response with the visible occluder (see above) is not due to a (forward) masking effect from the occluder, since the latter was absent in the invisible conditions. In fact, the net response levels of the visible and invisible conditions correlated well us- 
ing either moving (Spearman $R=0.72)$ or static $(R=0.63)$ occluding patterns. The differences in response latencies between the visible, invisible, and no-occlusion control conditions are given in Table 1 . In the case of the moving conditions, the latency in the invisible condition was significantly shorter than in the visible condition ( $42 \mathrm{msec}$; Wilcoxon matched pair test, $T=3.5 ; p<0.01$ ), while occluder visibility had only a small, nonsignificant effect on response latency with the static occluder. Overall, the response latencies of the two invisible conditions were similar to those of the no-occlusion condition (Table 1).

For all four occluder conditions, the average response declined with shape rank, indicating that the shape preference was similar to that observed in the no-occlusion condition (Fig. $14 A, B)$. For those units that remained significantly responsive in the visible occlusion condition ( 15 in both cases), we computed the average normalized response (see above for the procedure) as a function of the shape rank. As shown in Figure 14, $D$ and $E$, the shape selectivity curves are smoother when the occluder is visible than when it is not visible, but in both static and moving conditions, the interaction between occluder visibility and shape rank was not significant (ANOVA; $p>0.05$ ). Thus, occluder visibility only weakly affects the shape selectivity, if at all. On one hand, this agrees well with the similarity of the shape discrimination performance of monkey and man in visible and invisible conditions. On the other hand, the absence of a visibility effect for the cells suggests that shape completion is not required to obtain selective responses, since even shapes for which parts of the outline have been deleted, and which do not yield the percepl of a completed shape (the invisible conditions), are sufficient for the shape selectivity of these units.

Filled shapes. We tested the selectivity for filled shapes in noocclusion, the SV, and the SI occlusion conditions for 18 units. The average net response as a function of the shape rank, again determined using the responses to the nonoccluded shapes, is shown in Figure $14 C$. As with the outline shapes, there is a marked decrement in the average response with occlusion. Furthermore, the response latency was significantly shorter in the no-occlusion condition compared to the visible (mean difference, $20 \mathrm{msec}$ ) and invisible (mean difference, $21 \mathrm{msec}$ ) occluder conditions (Friedman ANOVA; $\chi^{2}=6.7 ; p<0.03$ ). These latency differences between the occlusion and no-occlusion conditions were similar to those observed for the static outline tests, but were only half the difference in response latency between the MV and no-occlusion outline conditions (Table 1). As for the static outlines, occluder visibility had no significant effect on neural response latency (Table 1).

The interesting finding is that the selectivity for the filled shapes, unlike shape outlines, can depend on the visibility of the occluding pattern. For a number of units, the responses for the shapes differed markedly between the two conditions, an example of which is shown in Figure 15. Note that the shape preference was similar in the no-occlusion and visible occlusion condition, while the response was absent when the same occlusion pattern was not visible. It should be stressed that the shape components presented in the SV and SI conditions are identical, the only difference is that the occluding pattern is also present in the visible condition. Thus, this cell does not merely respond to the shape components, since its response also depends on the presence of the occluder pattern.

In contrast to the outline shapes, the $\mathrm{SV}$ and SI responses showed no significant corrclation (Spcarman $R=0.01$ ) for the 18 cells tested. This lack of correlation implies that, as for the
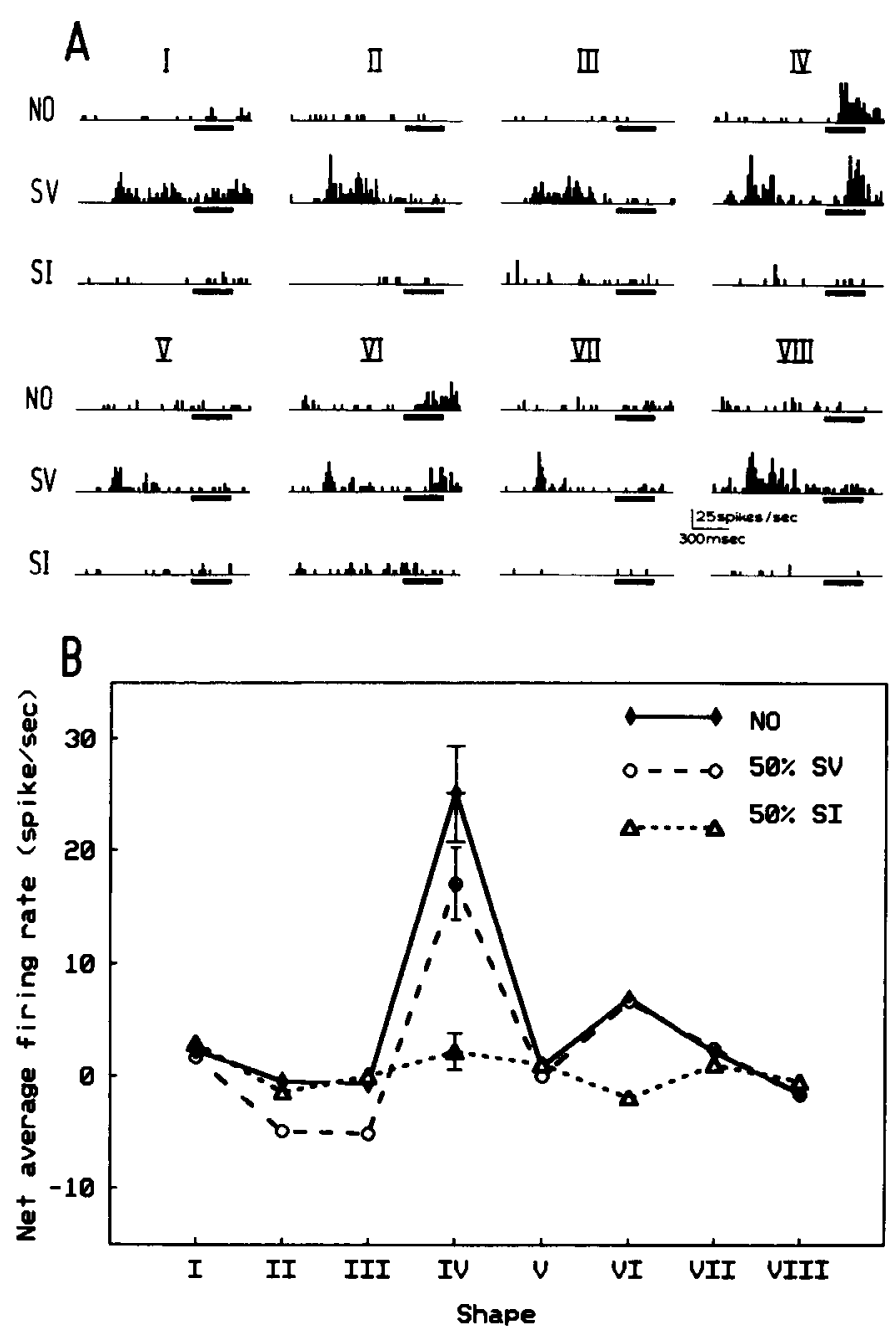

Figure 15. Effect of occluder visibility on the selectivity for filled shapes: example of one neuron (monkey 2). A, Peristimulus time histograms of the responses to the eight shapes in the no-occlusion ( $\mathrm{NO}$; 1st row of each shape), static visible occlusion ( $S V ; 2$ nd row), and static invisible occlusion ( $S I ; 3$ th row). Other conventions are the same as in Figure $5 A$. This neuron also responded to the occluder pattern itself (2nd row histograms). B, Average response for the eight different shapes in the no-occlusion $(N O)$, visible $(S V)$, and invisible $(S I)$ occluder condition for the same neuron as in $A$. Standard errors of the mean are indicated.

single unit of Figure 15, the responses to the filled shapes generally depended on the visibility of the occluder. Furthermore, in those units that responded significantly in the SV condition $(15 / 18$; Fig. $14 F)$, the shape selectivity in the SV condition was significantly different from that in the SI condition [ANOVA; $F(7,98)-2.48, p<0.02]$. The curve relating the average normalized response to the shape rank was flatter in the invisible condition (Fig. 14F), indicating that the shape selectivity was better maintained when the occluding pattern was visible than when it was not visible. Occluder visibility did not produce this effect on shape selectivity with the shape outlines (see above; Fig. 14E). Yet, in the invisible condition the filled shapes gave better responses to rank 1 than to rank 8 (Fig. 14C,F), indicating that some shape selectivity still remained. The latter may explain the performance of the subjects in this invisible condition: subjects were still able to discriminate the filled shapes when the 
shapes were occluded by the SI pattern, although not as well as in the other occluder and no-occlusion condition.

\section{Discussion}

The main findings of this study are as follows. (1) IT units remain selective for shapes that are partially occluded by another pattern. The responsiveness of the neurons, however, is affected by the occlusion: the greater the degree of occlusion, the less the response strength. This holds for shape outlines as well as filled shapes, i.e., shapes in which the border as well as the internal region differ in luminance from the background, and for static and moving occluder patterns. (2) The shape selective responses to partially occluded shapes require longer stimulus durations than nonoccluded shapes. This fits well with the results of a psychophysical experiment demonstrating better shape discrimination performance at short durations in the no-occlusion compared to the occlusion condition. (3) The effect of the occluder visibility on shape selectivity, as with human shape discrimination performance, was greater for filled shapes than for shape outlines.

Humans as well as the one monkey tested were able to discriminate the shapes under the partial occluding conditions used in this study. Furthermore, both species required longer stimulus durations to discriminate partially occluded shapes and showed similar discrimination performance in the MV and MI occlusion conditions. These results suggest that shape discrimination under conditions of partial occlusion are similar in humans and monkey, as does a recent systematic study of the discrimination of partially occluded shapes in the monkey (Osada and Schiller, 1994). Given this degree of similarity between monkey and human shape discrimination, we can assume that monkeys, like humans, also have difficulties in recognizing filled shapes that are partially obscured by an invisible occluder.

The basic finding of this series of experiments is that the shape selectivity of IT neurons is only weakly affected by partial occlusion. This may not seem remarkable given the ease with which we are able to recognize these shapes under such conditions. Nonctheless, sclectivity for shapes that are partially occluded by another visible pattern is, computationally, not a trivial task. The first step is to segment the features belonging to the shape from those belonging to the occluder pattern. In our case, the shape outlines had the same luminance as the occluding pattern, excluding luminance differences as a segmentation cue. However, the different onset times of occluder and shape creates a temporal asynchrony cue that can be a potent segmentation cue. In fact, informal observations have indicated that shape discrimination was much more difficult when the onset of SV occluder and shape coincided. In the case of the MV condition, the temporal asynchrony cue is ineffectual because of the motion of the occluder. Indeed, a mechanism detecting luminance onsets will be active throughout the full MV occlusion period, and, thus, cannot differentiate between the onset of the shape and the spatiotemporal changes of the occluding pattern. However, mechanisms differentiating motionless from moving patterns could provide segmentation of the static shape from the moving occluder. Given the recent demonstration of responses to shapes defined by relative motion in IT (Sáry et al., 1993), and, thus, direct or indirect motion input to IT, it is very plausible that segmentation of the occluded shape and occluder pattern may have been based on the differences in motion. This is in agreement with the longer neuronal response latencies in the MV condition compared to no-occlusion and the SV condition, since detection of differences in motion requires at least two frames. For both the MV and SV occluder patterns, other cues such as differences in the spatial frequency content of outline and occluder elements, or the presence of $\mathrm{T}$ junctions (von Helmholtz, 1903; Guzman, 1969; Cavanagh, 1987) may also have contributed to shape-occluder segmentation.

Our physiological data do not indicate whether the segmentation of shape and occluder occurs in IT or in visual areas afferent to IT. However, the fact that V2 units respond to illusory boundaries created by static occlusion cues (von der Heydt et al., 1984) suggest that these segmentation processes may occur or begin at early levels of the visual pathway. Also, a series of psychophysical studies on the role of occlusion in apparent motion (Shimojo and Nakayama, 1990a) and depth perception (Shimojo and Nakayama, 1990b; Shimojo et al., 1988) suggest that occlusion-related processing occurs at relatively early levels.

Once shape and occluder have been separated, two possibilities arise: either the neuron simply responds to the discrete shape elements, or the cell interpolates the connected elements into a whole ("Gestalt"), i.e., amodal completion. Our results with the shape outlines suggest that the IT units respond selectively to the disconnected shape elements, since the responses of the neurons were similar when the occluder is invisible or visible, the latter being the only condition of these two under which we perceive completed shapes. Thus, we propose that these IT units respond selectively whenever a sufficient number of the components of the preferred shape are present in their receptive field. Furthermore, the response strength depended on the degree of occlusion, suggesting that the more shape components present, the larger the response. This relationship between occluder density and response strength argues against the idea that the IT units represent only a completed shape, since, perceptually, one perceives completed shapes for the occluder densities lower than $90 \%$. Indeed, both the decrease in neural response with increasing occluder density and the absence of any effect by occluder visibility on the shape selectivity, suggests that the presence of uncompleted shape parts are sufficient, while amodal completion is not necessary, to obtain a shape selective response in these IT neurons. We cannot exclude the possibility that these IT neurons respond to parts of the shape outline instead of to the overall shape outline. Our results indicate that the greater the number of elements of this critical shape part present, the stronger the response, and, that the response of the neuron does not correlate with the presence or absence of perceptual amodal completion of that shape part.

Our results regarding the filled shapes are apparently in disagreement with those of the shape outlines, since we found a difference in responses between visible and invisible occlusion conditions for the filled shapes. However, there is a fundamental difference between the shape outlines and filled shapes when occluded by an invisible occluder. In the case of the outlines, parts of them are deleted, while in the case of the filled shapes, not are only parts deleted but new boundaries are also added, i.e., those borders common to the occluder and occluded surface (labeled $\mathrm{c}$ in Fig. 13C). These common boundaries, not present in the outline conditions, close the nonoccluded regions, and, thus, form new shape elements, which may explain response differences between the nonoccluded and invisible occlusion conditions when filled shapes are used. It also explains the difficulty one has in recognizing filled shapes that are partially occluded by an invisible pattern (Bregman, 1981; see Hummel and Biederman, 1992, for a similar argument). When the occlu- 
der is visible, borders belonging exclusively to the shape can be segregated from borders common to the occluder and to the shape. Thus isolated, these borders can produce a shape selectivity similar to that in the no-occlusion condition. In fact, all our findings can be explained assuming two principles: (1) the occluder pattern is segregated from the shape, isolating the contours belonging to the shape from those common to the occluder and the shape (i.e., the segmentation process discussed above), and (2) the IT units respond to the disconnected, segregated, shape elements. The more elements of the appropriate shape present, the better the response. Thus, our data can be fully explained without assuming the presence of any representation of amodal contours in I'I' (also see previous paragraph).

The physiological results from the shape selectivity experiments fit well with the psychophysical data from shape discrimination experiments with man and monkey: both behavioral discrimination and single-unit shape selectivity decreased with increasing occluder density and decreasing shape duration. Furthermore, subjects were still able to discriminate, albeit not perfectly, the shapes in the invisible occlusion condition, a performance matching the reduced, but still significant, shape selectivity of IT neurons in that occluding condition. These results agree with the hypothesis that IT plays an important role in the discrimination and recognition of shapes under the variety of conditions encountered in natural environments. Thus, the shape selectivity of IT units is largely invariant with changes in the position, retinal size, and defining visual cue, just as shape recognition is. This study adds another kind of invariance to this list: the invariance to partial occlusion of a shape by another pattern.

\section{References}

Biederman I, Cooper EE (1991) Evidence for complete translational and reflectional invariance in visual object priming. Perception 20: 585-593.

Biederman I, Cooper EE (1992) Size invariance in visual object priming. J Exp Psychol [Hum Percept] 18:121-133.

Bregman AS (1981) Asking the "what for" question in auditory perception. In: Perceptual organization (Kubovy M, Pomerantz JR, eds), pp 99-118. Hillsdale, NJ: Erlbaum.

Cavanagh P (1987) Reconstructing the third dimension: interactions between colour, texture, motion, binocular disparity and shape. Comput Vision Graphics Image Process 37:171-195.

Desimone R, Albright TD, Gross CG. Bruce C (1984) Stimulus selective properties of inferior temporal neurons in the macaque. $J$ Neurosci 4:2051-2062.

Gross CG, Mishkin M (1977) The neural basis of stimulus equivalence across retinal translation. In: Lateralization in the nervous system (Harned S, Doty R, Jaynes S, Goldberg L, Krauthamer G, eds). New York: Academic.

Gross CG, Rocha-Miranda CE, Bender DB (1972) Visual properties of neurons in inferotemporal cortex of the macaque. J Neurophysiol 35: $96-111$.

Guzman A (1969) Decomposition of a visual scene into three-dimen- sional bodies. In: Automatic interpretation and classification of images (Grasseli AC, ed), pp 243-276. New York: Academic.

Hummel JE, Biederman I (1992) Dynamic binding in a neural network for shape recognition. Psychol Rev 99:480-517.

Judge SJ, Richmond BJ, Chu FC (1980) Implantation of magnetic search coils for measurement of eye position: an improved method. Vision Res 20:535-538.

Kanizsa G (1979) Organization in vision: essays on Gestalt perception. New York: Praeger.

Kirk RE (1968) Experimental design: procedures for the behavioral sciences. Belmont, CA: Brooks-Cole.

Michotte A (1964) La perception de la casualite. Leuven: Leuvense Universitaire Pers.

Michotte A, Thines G, Crabbe G (1964) Les complements amodaux des structures perceptives. Studia psychologica. Leuven: Leuvense Universitaire Pers.

Osada Y, Schiller PH (1994) Can monkeys see objects under conditions of transparency and occlusion? Invest Ophthalmol Vis Sci 35:1664.

Regan D (1991) Spatial vision for objects defined by colour contrast, binocular disparity and motion parallax. In: Spatial vision (Regan D, ed), pp 135-178. London: Macmillan.

Regan D, Hamstra S (1991) Shape discrimination for motion defined and contrast defined form: squareness is special. Perception 20:315336.

Rolls ET, Baylis GC (1986) Size and contrast have only small effects on the responses to faces of neurons in the cortex of the superior temporal sulcus of the monkey. Exp Brain Res 65:38-48.

Sáry Gy, Vogels R, Orban GA (1993) Cue-invariant shape selectivity of macaque inferior temporal neurons. Science 260:995-997.

Sato T, Kawamura T, Iwai E (1980) Responsiveness of inferotemporal single units to visual pattern stimuli in monkeys performing discrimination. Exp Brain Res 38:313-319.

Schwartz EL, Desimone R, Albright TD, Gross CG (1983) Shape recognition and inferior temporal neurons. Proc Natl Acad Sci USA 80 $5776-5778$

Shimojo S, Nakayama K (1990a) Amodal representation of occluded surfaces: role of invisible stimuli in apparent motion correspondence. Perception 19:285 299.

Shimojo S, Nakayama K (1990b) Real world occlusion constraints and binocular rivalry. Vision Res 30:69-80.

Shimojo S, Silverman GH, Nakayana K (1988) An occlusion related mechanism of depth perception based on motion and interocular sequence. Nature 333:265-268.

Tanaka K, Saito H, Fukada Y, Moriya M (1991) Coding visual images of objects in the inferiotemporal cortex of the macaque monkey. J Neurophysiol 66:170-189.

Vogels R, Orban GA (1990) How well do response changes of striate neurons signal differences in orientation: a study in the discriminating monkey. J Neurosci 10:3543-3558.

Vogels R, Orban GA (1991) Quantitative study of striate single unit responses in monkeys performing an orientation discrimination task. Exp Brain Res 84:1-11.

Vogels R, Orban GA (1994) Activity of inferior temporal neurons during orientation discrimination with successively presented gratings. $\mathrm{J}$ Neurophysiol 71:1428-1451.

Vogels R, Kovács Gy, Orban GA (1993) Selectivity of macaque inferior temporal neurons for partially occluded shapes. Soc Neurosci Abstr 19:28.

von der Heydt R, Peterhans E, Baumgartner G (1984) Illusory contours and cortical neuron responses. Science 224:1260-1262.

von Helmholtz H (1903) Handbuch der Physiologischen Optik. Hamburg: Voss. 